located in the Emergency Service, 58.8\% had no current diagnosis of COVID-19, 64.7\% without medical comorbidity, 35.3\% without psychiatric comorbidity, $52.9 \%$ with suicide attempt as the main reason for consultation, $52.9 \%$ without regular use of medications, $88.2 \%$ with psychopharmacological treatment; $70.6 \%$ received a psychiatric interview intervention; Regarding symptoms, all presented interpersonal problems, impulsivity, emotional instability and inappropriate anger, while $58.8 \%$ had alteration of identity and $94.1 \%$ had suicidality. For personal variables, $82.4 \%$ had no family history, $88.2 \%$ had no history of abuse or trauma, $52.9 \%$ had a history of substance use, and $88.2 \%$ had no previous hospitalizations. Conclusion. The most of patients with BPD were young adults, women, single, from Villa El Salvador, catholics, completed secondary school, housewives, from Emergency, no diagnosis of COVID-19, without medical or psychiatric comorbidity, consulted for suicide attempt, without habitual use of medications, with indicated psychopharmacological treatment, a psychiatric interview was conducted, they had active symptoms, history of substance use and no family history, abuse or hospitalizations.

\section{Triple chronotherapy for the rapid treatment and maintenance of response in depressed outpatients: a feasibility and pilot randomised controlled trial}

David Veale ${ }^{1 *}$, Marc Serfaty $^{2}$, Clara Humpston ${ }^{3}$, Andriani Papageorgiou ${ }^{4}$, Sarah Markham ${ }^{5}$, John Hodsoll ${ }^{5}$ and Allan $\mathrm{H}$ Young ${ }^{6}$

${ }^{1}$ IoPNN King's College London, South London and Maudsley NHS Foundation Trust; ${ }^{2}$ UCL Division of Psychiatry; ${ }^{3}$ IoPPN King's College, Institute of Mental Health, University of Birmingham; ${ }^{4}$ South London and Maudsley NHS Foundation Trust; ${ }^{5}$ IoPPN King's College and ${ }^{6}$ IoPPN King's College, South London and Maudsley NHS Foundation Trust

${ }^{*}$ Corresponding author.

doi: 10.1192/bjo.2021.199

Aims. Triple chronotherapy (defined as sleep deprivation for 36 hours, followed by 4 days of advancing the time of sleep, together with daily morning bright light therapy for 6 months) has demonstrated benefits for the rapid treatment of depressive symptoms in 4 small, controlled trials of in-patients. Our aims were to test the feasibility of recruitment and delivery of triple chronotherapy for out-patients with depression.

Method. In a single blind trial, 82 participants were randomised to either triple chronotherapy or a control intervention. The primary outcome was Hamilton Depression Rating Scale 6 item (HAM-D6) at 1 week. Timings of observer ratings were baseline; 1 week; 2 weeks; 4 weeks; 8 weeks and 26 weeks after randomisation. Triple chronotherapy consisted of (a) Total sleep deprivation for 36 hours. On Day 1 patients were supported in a small group to stay awake at night with an occupational therapist, (b) Phase Advance of Sleep over 4 days. Phase Advance began after the first night of sleep deprivation, when they left the hospital at about $8 \mathrm{am}$ and were asked to go to bed earlier at about $5 \mathrm{pm}$ and rise at about $1 \mathrm{am}$. Their sleep and wake up times were then shifted 2 hours later on each of the following three days until they attained their usual bedtime again at about $11 \mathrm{pm}$. As a control for the triple chronotherapy, participants were given psychoeducation and written information on sleep hygiene. They were also given SomniLight amber light daily for 1 week in the morning.

Result. Participants in the triple chronotherapy group were able to stay awake for the planned thirty-six hours and $89.9 \%$ adhered to the plan of phase advance of their sleep over the following 4 days. We achieved our recruitment target with 60 participants having completed the trial within 13 months. There were no reported adverse side effects. We explored outcomes and found a significant difference between the groups for the HAM-D6 at week 1,8 and 26 . Response (>50\% reduction in symptoms) was achieved by $52 \%$ in the triple chronotherapy group compared to $18 \%$ in the control group at week 1 . This gradually increased to $70 \%$ achieving response in the triple chronotherapy group at week 26 compared to $22 \%$ in the control group.

Conclusion. Triple chronotherapy produced a significant and rapid benefit after 1 week in out-patients with depression that was sustained at 26 weeks. Further cost-effective trials with a larger clinical sample size are required.

Audit on the monitoring of metabolic side effects of antipsychotics in acute inpatient psychiatric units at Fieldhead Hospital

Stephanie Vel En Tial ${ }^{\star}$, Steve Curran and Adebayo Ikuyajesin

Fieldhead Hospital, South West Yorkshire Partnership Trust

*Corresponding author.

doi: 10.1192/bjo.2021.200

Aims. The current audit aims to assess the compliance with Prescribing Observatory for Mental Health (POMH-UK) guidance on monitoring of metabolic side effects of patients prescribed antipsychotics. Compliance was monitored to ensure that all patients prescribed continuing antipsychotics have their body mass index (BMI), blood pressure, blood glucose and lipids checked within the expected time limits of minimum once per year.

Background. Patients diagnosed with Schizophrenia rank amongst the worst of chronic medical illnesses in terms of quality of life. This may in part be due to the use of long term antipsychotic medications, in particular the use of atypical antipsychotics which have been increasingly associated with metabolic side effects including hypertension, weight gain, glucose intolerance and dyslipidaemia. These side effects are related to the development of both diabetes mellitus and cardiovascular disease and can lead to increased mortality and morbidity, affecting compliance and engagement to healthcare services. Despite the availability of clinical guidelines, monitoring and screening of metabolic side effects in patients prescribed antipsychotics continues to be suboptimal.

Method. The audit involved a review of electronic records relating to physical health monitoring of patients at two acute inpatient units from January-March 2019. Demographic and clinical variables were collected which included ethnicity, diagnostic grouping as well as current medications. Data were collected on evidence of screening for hypertension, BMI, blood glucose and lipids. Descriptive statistics were applied to study the clinical features of the sample and examine whether performance met clinical practice standard.

Result. The audit overall demonstrated partial compliance with POMH-UK guidelines with a total of 31 patients admitted on long term antipsychotics. Of these patients, $86 \%$ were prescribed atypical antipsychotics with $14 \%$ prescribed typical antipsychotics. Screening only occurred in $68 \%$ of patients for lipid profile with only $71 \%$ for BMI and $74 \%$ for blood glucose. Blood pressure had the highest compliance rate of $87 \%$ of patients being screened. Conclusion. Early identification and monitoring of complications from metabolic syndrome may decrease the risk of more serious health outcomes and improve patients' quality of life. However in clinical practice, standards are not always met in accordance with best practice recommendations. Requirement of a tailored guideline for physical health monitoring with weekly planned interventions as well as adequate training and awareness of 
healthcare staff is imperative to drive improvement and increase adherence rates.

\section{Identifying Risk Factors for Re-admission: A Service Evaluation from an Adult Inpatient Mental Health Unit}

Kirsty Ward ${ }^{\star}$ and Suveera Prasad

Rotherham, Doncaster and South Humber NHS Foundation Trust ${ }^{*}$ Corresponding author.

doi: 10.1192/bjo.2021.201

Aims. To identify risk factors for re-admission to an acute inpatient general adult mental health ward. There is need to ensure that mental health services adapt to the increasing demand for inpatient beds Method. We conducted a single centre retrospective analysis of electronic records of 85 discharges from an adult mental health unit from 4th March 2019 - 5th August 2019. We collected information on demographics, admission details, substance use, forensic history, diagnosis as per the International Classification of Diseases 10th Edition (ICD-10), and discharge details and compared two cohorts; those re-admitted within three months of discharge and those who were not. Odds ratio (OR), 95\% confidence intervals $(\mathrm{CI})$ and $\mathrm{p}$ values were calculated where possible.

Result. Among seventeen service users who were re-admitted within the three month period there were nine women and eight men. There was no difference in ethnicity, employment or marital status. The mean length of admission for those readmitted was 48.2 days (range 1-140 days) and 47.1 days (range 1-350 days) for those who were not readmitted. Certain features were more prevalent among the readmitted group including forensic history $(58.8 \%$ [10] vs $26.5 \%$ [18], OR 3.97, CI 1.31-11.9, p value 0.007 ), substance misuse history (70.6\% [12] vs 55.9\% [38], OR 1.89, CI 0.60-5.97, p value 0.138$)$, previous contact with mental health services $(100 \%$ [17] vs 76.5\% [52]) and the rate of detention under the Mental Health Act at point of admission (76.5\% [13] vs $66.2 \%$ [45], OR 1.66 , CI $0.49,5.67$, p value 0.209 ).

Among those readmitted, a diagnosis of emotionally unstable personality disorder $(17.6 \%$ [3] vs $10.3 \%$ [7], OR $1.87, \mathrm{CI}$ $0.43,-8.14$, p values 0.203$)$ and substance misuse disorder $(41.2 \%$ [7] vs $17.6 \%$ [12], OR 3.27, CI 1.04-10.31, p value 0.218 ) were more prevalent. They were more likely to use illicit substances whilst they were an inpatient (23.5\% [4] versus $7.6 \%$ [5], OR 3.88, CI $0.92-$ 16.43 , $\mathrm{p}$ value 0.033$)$ and to be involved in police incidents $(35.3 \%$ [6] versus $17.6 \%$ [12], OR 2.55, CI 0.79-8.23, p value 0.059 ).

Conclusion. Our trends demonstrate that people with substance misuse, emotionally unstable personality disorder and forensic history are more likely to be readmitted to an adult mental health inpatient unit. They were more likely to misuse illicit substances and be involved with police during admission.

Evaluation of a novel consultant psychiatric clinic in general practices and its effects on secondary mental health contact and the general practitioners' perspectives

Kamran Mahmood ${ }^{1 \star}$, Daniel Whitney ${ }^{2}$ and Guy Brookes ${ }^{1}$

${ }^{1}$ Leeds and York Partnership Foundation Trust and ${ }^{2}$ Tees Esk and Wear Valleys NHS Foundation Trust

${ }^{\star}$ Corresponding author.

doi: 10.1192/bjo.2021.202
Aims. To assess whether direct access to a 45 minute screen appointment in a Consultant Psychiatric clinic, based in General Practice, affects; the number of contacts patients have with secondary care pre and post being seen; whether the General Practitioner (GP) would have referred to secondary services if the clinic had not been in operation; the GPs' views on how helpful the clinic was in understanding the patients' problems and managing the problems outside of secondary care.

Background. A Consultant Psychiatrist in Leeds offered bespoke 45 minute screening appointment clinics in three sister GP practices, accepting direct referrals from GPs without requiring referrals to the local Community Mental Health Team (CMHT). This model was created to reduce the number of patients moving repeatedly between GP and secondary mental health services as this was leading to patient dissatisfaction and increased GP and CMHT workloads.

Method. We compared the number of mental health contacts (per month), for each of the 57 patients who had been referred to the clinic, in the months pre and post being seen in the clinic. We also asked the involved GPs to complete a brief survey for each patient who had been referred to determine whether, they would otherwise have been referred to the CMHT and whether the clinic has helped with their understanding and management of the patients' problems.

Result. The mean number of contacts with secondary services before being seen in clinic was 3.30 per month compared to 0.44 after being seen. The mean difference of 2.86 is statistically significant on a paired-test with a P Value of 0.0149 (95\% confidence intervals of 0.58 to 5.13). We received 22 survey responses from GPs of patients referred to the clinic including for patients who did not attend. All 22 responses indicated that the patient would have been referred to the CMHT if the clinic had not been available. $95 \%$ were rated as being very helpful or moderately helpful in understanding the patient's problems. $91 \%$ were rated as very helpful or moderately helpful in managing the patients' problems outside secondary care.

Conclusion. Our evaluation has demonstrated that a model of direct access for GPs to a Consultant Psychiatric clinic can reduce referrals and patient contacts with secondary mental health services. GPs have found this model helpful in understanding patients' problems and managing the problems outside of secondary care.

\section{A retrospective case-control service evaluation of} CAARMS scores of patients with autism in York EIP, compared to age matched controls

Daniel Whitney ${ }^{*}$ and Stephen Wright

Tees Esk and Wear Valleys NHS Foundation Trust

${ }^{*}$ Corresponding author.

doi: 10.1192/bjo.2021.203

Aims. Studies show the prevalence of Autism Spectrum Conditions in Early Intervention in Psychosis (EIP) populations is $3.6-3.7 \%$, compared to approximately $1-1.5 \%$ in the general population. The CAARMS (Comprehensive Assessment of At Risk Mental States) is a national tool used by EIP services as a screening tool to bring patients into services and stratify their symptoms to determine what pathway may be most appropriate (First Episode Psychosis pathway (FEP) or At Risk Mental State pathway (ARMS)). As far as we are aware the CAARMS has not been validated in an autistic population. It is our view that several of the questions in the CAARMS may be interpreted differently by people with autism, thus affecting the scores. The aim 